\title{
ЗНАЧЕНИЕ АКТИВНОСТИ РЕНИН-АЛЬДОСТЕРОН-АНГИОТЕНЗИНОВОЙ СИСТЕМЫ В ПАТОГЕНЕЗЕ ГИПОКАЛИЕМИИ ПРИ COVID-19
}

Циберкин А.И., Головатюк К.А., Быкова Е.С., Каронова Т.Л., Андреева А.Т., Вашукова М.А., Цой У.А., Гринева Е.Н.

\author{
НМИЦ им. В. А. Алмазова» Минздрава России, Санкт-Петербург
}

Гипокалиемия - одно из частых электролитных явлений среди госпитализированных пациентов c COVID-19. Возможной причиной гипокалиемии при COVID-19 может быть гиперактивация ренин-ангиотензин-альдостероновой системы (РАAС).

ЦЕЛЬ: оценить активность РААС при различном уровне калия крови у пациентов, госпитализированных с пневмонией на фоне COVID-19.

МАТЕРИАЛЫ И МЕТОДЫ: В поперечное когортное исследование включили 172 пациента с пневмонией, вызванной вирусом SARS-CoV-2. У 77 пациентов определили показатели калия, альдостерона и ренина в венозной крови. Кроме того, оценивали уровни острофазовых показателей, степени поражения легочной ткани, тяжесть COVID-19 и исходы госпитализации между группами пациентов с гипокалиемией и нормальным уровнем калия.

РЕЗУЛЬТАТЫ: гипокалиемию выявили у 19 из 77 пациентов (25\%): медиана уровня калия крови у пациентов с гипокалиемией и без составила 3.1 [2.8-3.3] и 4.1 [3.9-4.5] ммоль/л соответственно ( $\mathrm{p}=0.001)$. Уровни альдостерона и ренина плазмы у пациентов с гипокалиемией и без достоверно не различались: альдостерон 76.0 [57.7-121.5] и 70.9 [26.3-113.8] пг/мл ( ( $p=0.352$ ) соответственно. Значимых различий в степени поражения легочной ткани, показателях, характеризующих выраженность системного воспаления, тяжести COVID-19, длительности госпитализации и смертности между пациентами с выявленными электролитными нарушениями и без также не обнаружили.

Выводы: в ходе настоящего исследования не было выявлено лабораторных признаков повышения активности PAAC у больных с COVID-19 и выявленной гипокалиемией. Уточнение генеза и значимость гипокалиемии среди пациентов с COVID-19 требует дальнейших исследований. 